\title{
A localization-independent approach for invisible and impalpable ground-glass opacity nodules detection in an in vitro lung specimen: two case reports
}

\author{
Bei Qing^, Zhenkun Xia, Wei Wang, Linguo Gu, Hongzuo Chen, Yunchang Yuan \\ Department of Thoracic Surgery, the Second Xiangya Hospital, Central South University, Changsha, China \\ Correspondence to: Yunchang Yuan. Department of Thoracic Surgery, the Second Xiangya Hospital, Central South University, 139 Middle Renmin \\ Road, Changsha 410011, China. Email: yuanyc@csu.edu.cn.
}

\begin{abstract}
A growing number of ground-glass opacity (GGO) nodules are screened out in lungs. Small GGOs are frequently neither visible nor palpable, thus undetectable during operation. Various nodule localization techniques have been developed to facilitate the intraoperative detection of GGO nodules; however, general localization techniques are infeasible or inappropriate in some cases. The detection of small GGO is a great challenge, even within a surgical specimen in the absence of preoperative localization. A localization-independent approach for GGO detection is urgently needed. Herein, we report two cases with invisible and impalpable small GGO which were not appropriate for preoperative localization. The lesions were anatomically resected under the guidance of three-dimensional (3D) reconstruction and got an adequate margin distance. A vessel (artery, vein, or bronchus) which had advanced into or immediately adjacent to the nodule was assigned as a reference vessel. By dissecting and tracing the reference vessel from proximal to distal, the GGO lesions were successfully detected in the surgical specimens, to the eventual obtainment of an accurate pathological diagnosis. Via the two case reports, we introduced an easily handled approach, namely dissecting and tracing a reference vessel, for GGO detection. The novel approach was first described. Combined with precise anatomical segmentectomy guided by $3 \mathrm{D}$ reconstruction, it provides an alternative scheme for GGO resection with no need for preoperative localization.
\end{abstract}

Keywords: Ground-glass opacity (GGO); localization; three-dimensional reconstruction (3D reconstruction); segmentectomy; case report

Submitted Sep 10, 2021. Accepted for publication Oct 14, 2021.

doi: 10.21037/atm-21-4966

View this article at: https://dx.doi.org/10.21037/atm-21-4966

\section{Introduction}

The widespread application of low-dose computed tomography (LDCT) screening and extensive implementation of lung cancer screening programs have substantially increased the detection rate of ground-glass opacity (GGO) lesions (1). Persistent GGO is more likely to be malignant. Ye et al. reported that 1,070 of the 1,155 (92.6\%) GGO lesions turn out to be malignant after resection (2). Young female nonsmokers were found to get a high GGO detection rate, which may relate to risk factors such as cooking fumes, second-hand smoking, hormones, air pollution. While the exact etiology of GGO remains to be clarified (3). Video-assisted thoracoscopic surgery (VATS) resection remains the first treatment option for GGO. However, small subpleural GGOs are frequently neither visible nor palpable, and due to their low density and small size, the detection of nodules during the VATS resection process presents quite a challenge for surgeons. To overcome those challenges, various techniques have been designed to mark small pulmonary nodules (SPNs) during VATS resection, including hook wire (4) or micro coils placement (5),

$\wedge$ ORCID: 0000-0003-4740-2923. 
methylene blue (6) or tissue adhesive injection (7), and so on. Although the success rates of the abovementioned methods have been reported as fair, most of those techniques are invasive, posing the risk of complications like pneumothorax, hemothorax, and pleural pain. Moreover, some locations (such as in the apex of the lung, behind the scapula, and so on) are technically infeasible for those locating techniques (8). Three-dimensional (3D) reconstruction simulation allows precise visualization of pulmonary arteries, veins, bronchus, and target lesions, while revealing anatomical variations (9). Yang et al. raised that 3D reconstruction is an efficient and non-parenchymal injury tracing method for GGO lesions' location while undergoing thoracoscopic segmentectomy (10). Under the guidance of $3 \mathrm{D}$ reconstruction, precise anatomical segmentectomy resection, including subsegmentectomy or the combinations, signifies a new direction for resection of GGOs independent of localization techniques.

Without nodule localization, it is a huge challenge for both the surgeon and pathologist to detect invisible or impalpable GGOs even with an in vitro surgical specimen. The failure of detection may ultimately lead to no pathological confirmation which will affect the subsequent treatment decision. Moreover, doubts may arise regarding whether the nodule had been resected or not when it is close to the cut edge. This lack of clarity would cause great psychological stress to both doctors and patients. Hence, an accurate and easily handled method to detect invisible and impalpable GGOs within in vitro surgical specimens is urgently needed. Visa the following two cases, we will introduce a novel approach, that is dissecting and tracing a reference vessel, to detect GGOs in the surgical specimen after anatomical resection. Our approach, combined with precise anatomical resection, presents an alternative, supplementary, and sometimes remedial method to deal with invisible or impalpable GGOs. It was routinely utilized in our surgical group for deep impalpable GGOs, and was successfully performed in about 40 cases. To the best of our knowledge, this is the first time that this approach has been described. We present the following cases in accordance with the CARE reporting checklist (available at https:// dx.doi.org/10.21037/atm-21-4966).

\section{Methods}

The preoperative high-resolution computed tomography (HRCT) data (contrast-enhanced CT with a slice thickness of $1.0 \mathrm{~mm}$, DICOM standard format) was obtained from an imaging workstation in our hospital. The data was sent to
Qingdao Hisense Medical Equipment Co., Ltd. (Qingdao, China) for $3 \mathrm{D}$ reconstruction simulation and was then used for the construction of a surgical plan. Usually, for deep and impalpable nodules, vessel enfolding, meaning that that the lesion is enfolded by vessels (artery, vein, or bronchus) or that there is at least 1 vessel between the target nodule and cutting plane, is mandated to ensure that the nodule is resected with an adequate margin distance. A relatively superficial vessel which had advanced into or was immediately adjacent to the nodule was assigned as a reference for the localization. Under the guidance of $3 \mathrm{D}$ reconstruction, the surgery proceeded as usual (11). The central part of the intersegmental plane was dissected by an electric or ultrasonic scalpel, while the peripheral part was cut by staplers. After the specimen had been removed from the thoracic cavity, palpation was the first method used to detect the nodule. If it was deemed a failure or indeterminate, we did not incise the specimen for fear of corrupting the anatomical integrity; instead, the selected reference vessel was carefully dissected and traced from proximal to distal. The lung tissue between the pleura and the reference vessel was incised to expose the vessel. Every time we encountered branches, repeated matching with the $3 \mathrm{D}$ reconstruction image was performed to select the right bifurcation to trace forward. For the nodule that the absent reference vessel advanced into, the direction to cut the lung tissue should be thoroughly considered to avoid the nodule being dissected in the cut surface. By taking these measures, the nodule size cannot be artificially reduced, which may increase the difficulty of nodule localization. With the assistance of a $3 \mathrm{D}$ reconstruction image, there is no difficulty in identifying whether the nodule adjacent to the reference vessel is on the right or left side. As the anatomy nears the target area, the target nodule could be easily found by incising the lung tissue adjacent to the reference vessel. Meanwhile, for the nodule that the reference vessel advanced into, the target lesion will be exposed as we dissect along the reference vessel. The nodule is then marked with a suture and sent for frozen-section examinations. Intraoperative frozen pathology determines the remainder of the surgery.

\section{Case presentation}

\section{Case 1}

A 63-year-old female patient was admitted to our department for treatment of multiple nodules in the right lung. She did not feel any discomfort and had no specific history of malignant tumors. The HRCT scan revealed a 

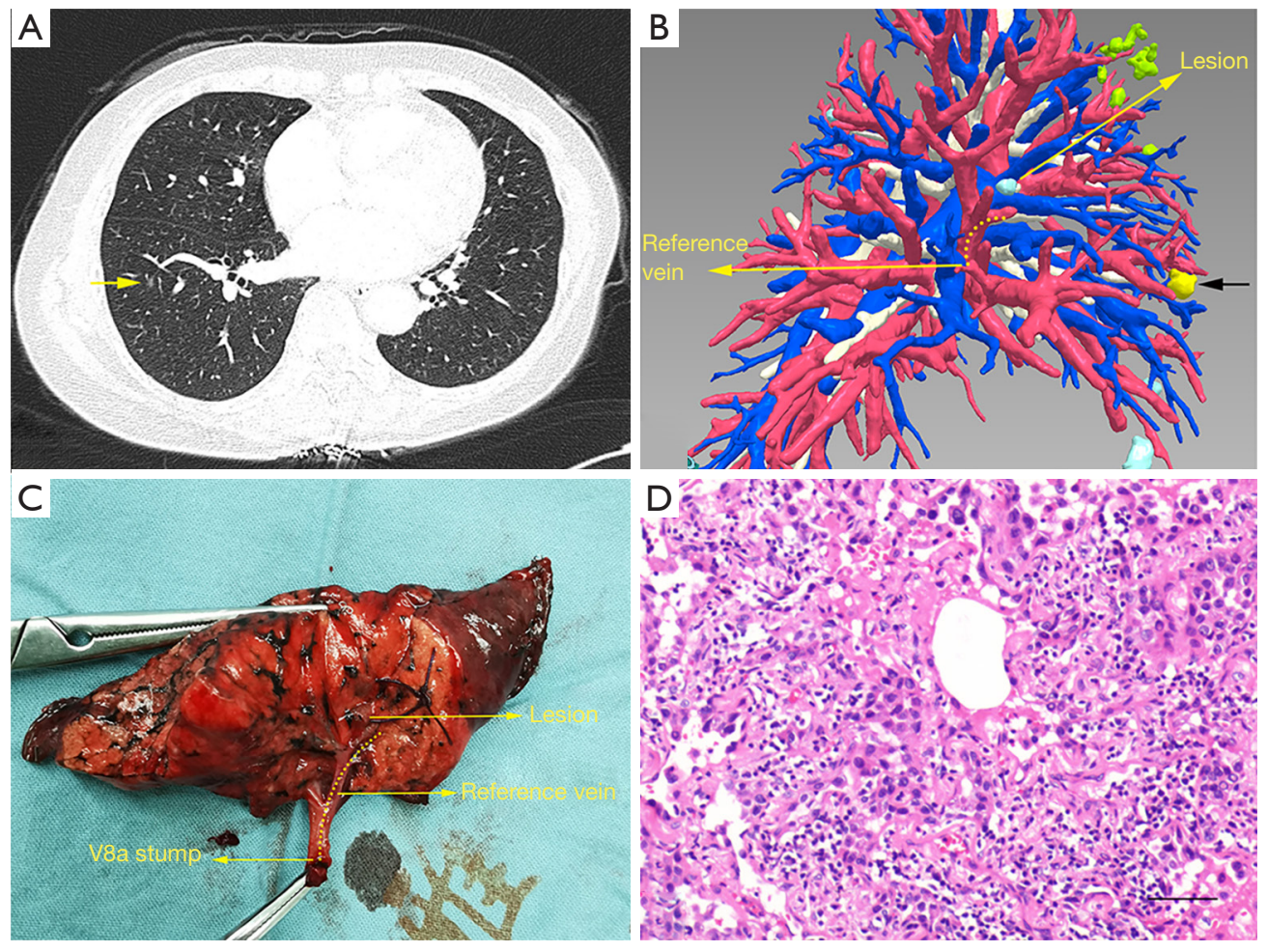

Figure 1 CT scan image, 3D reconstruction, surgical specimen and paraffin section of the GGO lesion in RS8a. (A) The CT scan shows the smaller GGO lesion in the right S8a; the yellow arrowhead indicates the location of the GGO. (B) The 3D reconstruction image of lung, the right V8a vein is selected as the reference vessel, the GGO lies just on the left of its branches. The black arrowhead indicates the bigger GGO in the right middle lung. (C) In vitro specimen of subsegmentectomy, V8a vein was carefully dissected and traced, the target GGO is detected just beside one of the branches of V8a vein. (D) The smaller GGO lesion was diagnosed as adenocarcinoma in situ (hematoxylin-eosin staining, $\times 100$, scale bar, $100 \mu \mathrm{m})$. CT, computed tomography; 3D, three-dimensional; GGO, ground-glass opacity.

$10 \mathrm{~mm}$ GGO (CT value: -862 to $-368 \mathrm{HU})$ in the right middle lobe and another $5 \mathrm{~mm}$ GGO (CT value: -834 to $-357 \mathrm{HU})$ in the right lower lobe. The preoperative 3D reconstruction showed that the $5 \mathrm{~mm}$ GGO in the right lower lobe was located in the S8a subsegment and that the nodule lay adjacent to the left of the branch of V8a. Relevant preoperative examinations found no apparent contraindications. Right middle lobectomy and right lower S8a subsegment resection were proposed. Under general anesthesia and single-lung ventilation, the left decubitus position was taken. A $1 \mathrm{~cm}$ incision was made in the midaxillary line of the 7 th intercostal space on the right side as an observation hole. A $3 \mathrm{~cm}$ incision was made in the anterior axillary line of the 4th intercostal space on the right side as an operation hole. During the operation, the right middle lobe was removed at first, and the S8a subsegment resection of the right lower lobe followed. After dissociation and transection of the A8a artery, B8a bronchus, and V8a vein, the intersegmental plane was confirmed by the inflation-deflation method. The central part of the intersegmental plane was dissected by an electric or ultrasonic scalpel, while the peripheral part was cut by staplers. Then, the S8a subsegment was removed, and the lesion was neither visible nor palpable in the surgical specimen. The V8a was selected as a reference, and the segment was incised from the direction of oblique fissure. After dissecting and tracing about three branches, the lesion was found just beside the left side artery (Figure 1). The resection margin was verified as reasonably sufficient. Intraoperative pathology confirmed that the bigger nodule was micro-invasive adenocarcinoma, while the smaller nodule was adenocarcinoma in situ. The postoperative paraffin pathological results were consistent. A chest $\mathrm{X}$-ray on the second day after surgery showed a good lung re-expansion. The patient recovered smoothly and was discharged on the third day after surgery. 

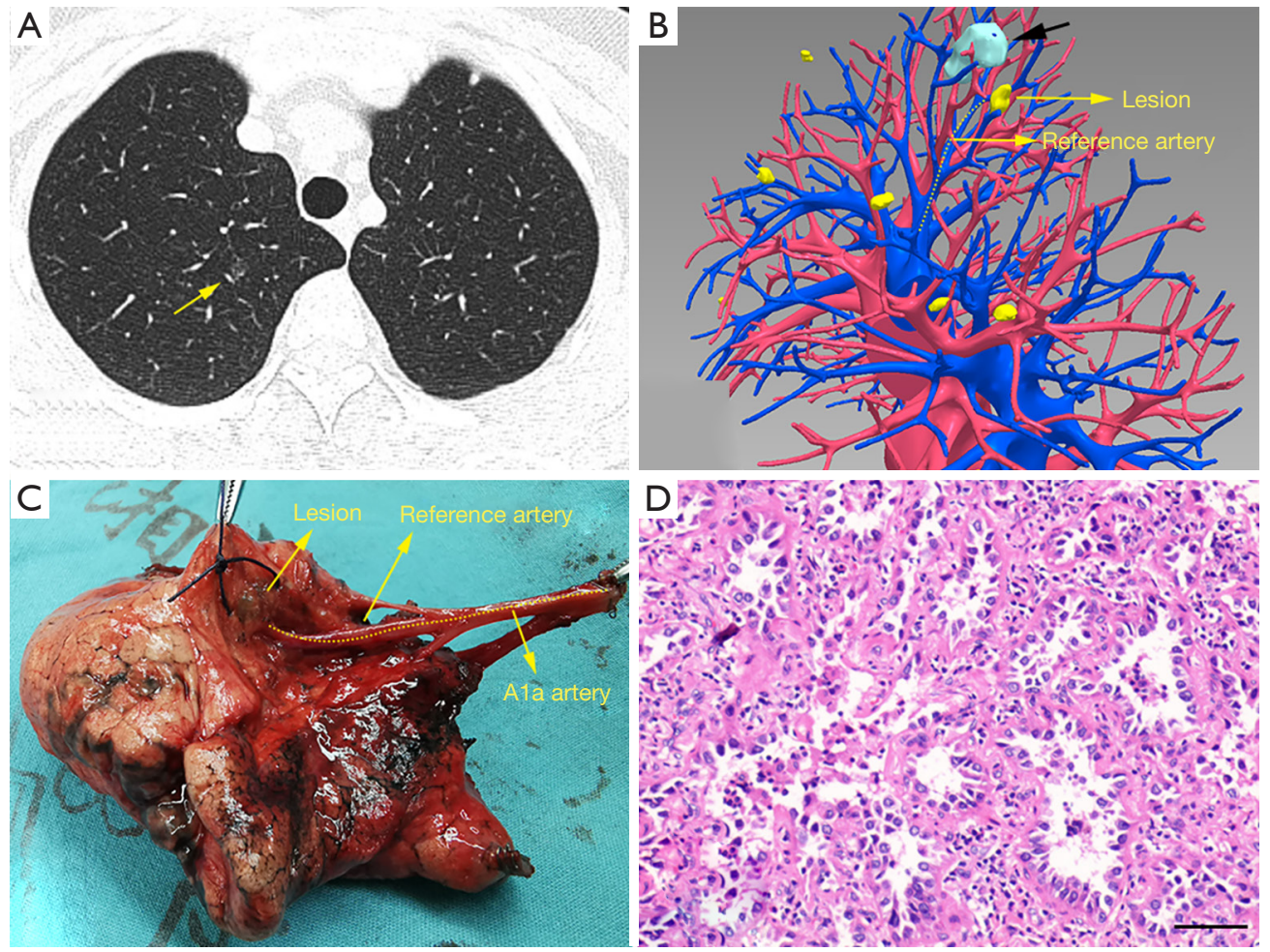

Figure 2 CT scan image, 3D reconstruction, surgical specimen and paraffin section of the GGO lesion in RS1. (A) The CT scan showed the smaller GGO lesion in the right S1; the yellow arrowhead indicates the location of the GGO. (B) The 3D reconstruction image of lung, the right Ala artery was selected as the reference vessel, the GGO lies just on the right of its branches. The black arrowhead indicates the bigger GGO in the same segment. (C) In vitro specimen of segmentectomy, A1a artery was dissected and traced, the target GGO is detected just beside one of the branches of A1a artery. (D) The smaller GGO lesion was diagnosed as adenocarcinoma in situ (hematoxylin-eosin staining, $\times 100$, scale bar, $100 \mu \mathrm{m})$. CT, computed tomography; 3D, three-dimensional; GGO, ground-glass opacity.

\section{Case 2}

A 48-year-old female patient was admitted to our department for treatment of a lesion in RS1. She did not feel any discomfort and had no specific history of malignant tumors. The HRCT scan found a $10 \mathrm{~mm}$ GGO (CT value: -875 to -392 HU) and a $5 \mathrm{~mm}$ GGO (CT value: -890 to $-396 \mathrm{HU}$ ). The $3 \mathrm{D}$ reconstruction identified that the GGOs were all located in the RS1 and the smaller lesion was on the right side of the Ala branches. Relevant preoperative examinations found no obvious contraindications. A right upper lobe S1 segment resection was planned. During the operation, the position was the same as that of case 1 , and the $A 1 a+b$ artery and B1 bronchus were divided and resected. After dissociating and severing V1a, The S1 segment was removed using the same method as in case 1 . The bigger lesion was then detected by palpation, while the smaller lesion was impalpable. Under the guidance of $3 \mathrm{D}$ reconstruction, the
A1a artery was selected as a reference. We cut the in vitro segment from the mediastinal surface of the lung. After dissecting and tracing about three branches, the lesion was found just beside the right side of the artery (Figure 2). The resection margin was verified as reasonably sufficient. Intraoperative pathological results confirmed that the bigger nodule was micro-invasive adenocarcinoma, while the smaller nodule was adenocarcinoma in situ, and the postoperative paraffin pathological results were consistent. The chest X-ray on the second day after surgery showed a good lung reexpansion. The patient was then discharged on the third day after the surgery.

\section{Ethical statement}

All procedures performed in this study involving human participants were in accordance with the Declaration of Helsinki (as revised in 2013). The study was approved by 
Medical Ethics Committee of the Second Xiangya Hospital of Central South University (No. 2019022). Written informed consent was obtained from the patient for publication of this case report and accompanying images. A copy of the written consent is available for review by the editorial office of this journal.

\section{Discussion}

With the development of operative instrumentation and techniques, VATS has been increasingly widely used in thoracic surgery all over the world (12-14). Its perioperative safety and long-term oncological efficacy for non-small cell lung cancer (NSCLC) have been demonstrated by several clinical trials and meta-analyses. Moreover, the minimally invasive nature of VATS delivers numerous advantages, namely, less severe postoperative complications, less postoperative pain, faster recovery, and more esthetic appearance (15-20). Along with the popularization of HRCT, a growing number of GGO lesions have been detected in lungs (21). Due to the advantages of minimally invasive operation, VATS has become the most common approach to diagnose and treat GGOs. The challenge of intraoperative location of these low-density and smallsize nodules, which are primarily invisible and impalpable, makes their diagnosis and treatment difficult. Another considerable challenge is the smooth implementation of the VATS. To solve these problems, various methods have been developed for the localization of SPNs (7,8,22-24).

Hook wire placement is the oldest method for localization of SPNs. It evolved from the conventional mammographic needle and is currently widely used (25). The hook wire not only indicates the location of pulmonary nodules but also aids the resection if gently retracted (26). A meta-analysis reported the localization success rate (without dislodgement or migration) of hook wire as $93.9 \%$ [95\% confidence interval (CI): 0.91 to 0.96 ] (27); however, the relatively high complication rate is a major limitation of this technique. Pneumothorax, pulmonary hemorrhage, hemothorax, and pleural pain are the most common complications of hook wire placement (28). Other percutaneous puncture-based techniques, such as micro coil placement (5), injection of dye materials (6), medical adhesive injection (7), contrast medium localization (29), radiotracer guided localization (30), and so on were also reported to be feasible. Complications are consistently associated with percutaneous lung punctures and lead to bad treatment experiences for patients. While hook wire placement has a long history, intraoperative ultrasonographic localization was proposed not long afterwards (24); however, this technique has not been as popular mainly due to it being operator dependent and requiring complete collapse of the assessed lung (31). Many new techniques have been developed in recent years, including electromagnetic navigation bronchoscopy (ENB) localization $(32,33)$ and $3 \mathrm{D}$ printing localization $(34,35)$. Although these methods have reportedly achieved reasonably fair success rates, operational complexity and special equipment dependence may impede their general use. Taking the drawbacks of those localization techniques into consideration, the concept of precise resection of GGOs with no need for localization warrants more attention.

The localization of pulmonary nodules is mainly conducted to facilitate wedge resection. Although wedge resection is a relatively simple and effective procedure for peripheral GGOs, there are many cases in which wedge resection is inappropriate or infeasible. First, for tumors deep in the parenchyma, wedge resection has difficulty obtaining adequate margin, therefore, it is generally considered problematic. Koike et al. (36) concluded that wedge resection is frequently associated with margins less than $1 \mathrm{~cm}$ and a high risk for locoregional recurrence. Segmentectomy appears to be a better therapeutic choice. Besides, the traditional percutaneous puncture-based techniques become technically infeasible when the nodules are located certain places, including in the apex of the lung, behind the scapula, and close to the mediastinal heart or great vessels (4). Wedge resection is blind and imprecise without nodule localization. There are so many possibilities to take into consideration including accidental interoperative encounter of previously undetected lesions, dislodgement or migration after a preoperative localization, or the absence of a plan for preoperative localization. A remedial conversion to precise anatomical resection would be more reliable and accurate. Given the cases for which wedge resection is inappropriate (or infeasible) and the shortcomings of localization techniques, we advocate an alternative scheme, namely, precise segmentectomy (including subsegmentomy and the combinations) guided by $3 \mathrm{D}$ reconstruction. Precise segmentectomy can precisely resect the lesion independent of marking while ensuring sufficient margins, offering an alternative, remedial, and sometimes more appropriate choice for GGOs.

The VATS pulmonary segmentectomy is technically meticulous, considering the anatomical vascular or bronchial variations and the difficulty of confirming the location of nodules and target segments. The $3 \mathrm{D}$ reconstruction allows 
precise visualization of pulmonary arteries, veins, bronchus, and target lesion, reveals anatomical variations, displays the relative position between the target nodule and vessels and the target segment $(9,37,38)$. The $3 \mathrm{D}$ reconstruction enhances precision of segmentectomy, facilitates the location of nodules, and shortens the learning curve for surgeons. Its effectiveness to guide the resection for SPNs via segmentectomy has been generally accepted. Kato et al. (39) performed thoracoscopic segmentectomy for 58 patients with invisible and impalpable SPNs using 3D reconstruction rather than preoperative localization. All nodules were correctly resected without nodule marking. Xue et al. (40) analyzed 68 patients with small $(\leq 2 \mathrm{~cm})$ GGOs which were successfully resected by VATS segmentectomy without preoperative marking. They reported that $3 \mathrm{D}$ reconstruction is very convenient for pulmonary nodules and target segment locating and is helpful for precise resection of GGO lesions. Chan et al. (41) demonstrated that all 33 participants achieved a correct tumor segmental localization in the guidance of $3 \mathrm{D}$ reconstruction. Sufficient resection margins are critical for the oncological efficacy of segmentectomy (42-44). The $3 \mathrm{D}$ images guide the surgeon in formulating suitable plan to ensure the resection margin. Wu et al. (45) reported that all cases in the precision (3D) group had an adequate margin, while $4(7.3 \%)$ participants in the routine (non-3D) group had inadequate margins. Xue et al. (40) demonstrated that the $3 \mathrm{D}$ reconstruction groups all had adequate resection margin, while the rate in the non-3D group was $87 \%$. They also recorded 7 of 36 participants' resection extents were adjusted after 3D reconstruction. Here, we introduced our approach to ensure the margin for a deep lesion. Vessel enfolding, as described earlier, is mandated as we make the surgical plan under the guidance of $3 \mathrm{D}$ reconstruction. The selected vessel has two advantages in the surgical procedure. Firstly, as we know, the lung tissue is fragile and ductile. The energy device can quite easily deviate from the ideal plane when we separate the central part of the intersegmental plane, resulting in the inadequacy of the resection margin. The vessel is much tougher than the lung tissue which serves as an obstacle to prevent the energy device from getting too close to the tumor. Secondly, the vessel and its branches distribute in the lung parenchyma like a tree. After being transected, its stump could be a hauling point to pull the target segment tissue away from the intersegmental plane as we cut the plane. While for a superficial nodule, a deviation away from the target segment when we cut the intersegmental plane with a stapler is consistently practical for the margin guarantee.

It is also a challenge when small and low-density GGO lesions are detected in the in vitro specimen after being successfully resected. Missing lesions, though rarely reported, occasionally occur in the real world. Ji et al. (46) failed to locate two pure GGOs among 43 surgical specimens, even with the guidance of $3 \mathrm{D}$ reconstruction/print. The failure of localization may ultimately lead to no pathology confirmation and affect the scope of a given operation and the subsequent treatment strategy. Moreover, we may be unsure whether the nodule had been resected or not when the lesion is close to the cut edge. Such a lack of clarity is stressful for both doctors and patients. Thus, it is urgent to find a reliable method to detect invisible and impalpable GGOs in surgical specimens.

Li et al. (47) introduced a CT-guided needle localization for GGOs in postoperative specimens, while the operational complexity and the requirement of CT scanner and inflatable aerator limit its widespread use in clinical practice. Kato et al. (39) performed thoracoscopic segmentectomy using 3D CT simulation without nodule marking for nonpalpable and non-visualized SPNs; although all the lesions were resected, they did not mention the method for nodules detection in the surgical segment. Ji et al. (46) conducted a prospective cohort study evaluating the effectiveness of $3 \mathrm{D}$ reconstruction/print for locating multiple nodules in specimens after surgery. Some 124 of 126 (98.4\%) nodules were successfully located. The $3 \mathrm{D}$ reconstruction/print acted as a guide to determine the location and relative position of nodules. Again, they did not declare the exact method by which they located nodules. Moreover, there were two pure GGOs that remained undetected. In this study, we presented an accurate and easily implemented approach for addressing the issue of detecting invisible and impalpable GGOs in vitro specimens by requiring no other localization techniques. The two nodules in the two cases which were detected by our approach were pure GGOs with a diameter of $5 \mathrm{~mm}$. Their CT value was relatively low, with a range of about -900 to $-300 \mathrm{HU}$. Their successful detection, to a certain extent, revealed the effectiveness of our approach. The approach was performed in about 40 cases in our surgical group. Only one case $(2.5 \%)$ with GGO lesion in the RS4 failed for the reason that the lung was incised before tracing, which corrupted the integrity of reference vessel. However, an additional cohort study is required to assess the effectiveness and safety of this method. We are collecting more cases and data for further evaluation.

Precise anatomical resection is the base and precondition of our approach. The 3D reconstruction and simulation allow precise visualization of pulmonary arteries, veins, 
bronchus, and target lesions, reveals the relative position of the target nodule and vessels and the target segment, which is vital for preoperative planning. When planning preoperatively, we should emphasize the concept of lesion and its margin rather than the conventional and standard concept of segment or intersegmental plane. We have presented a new idea, the "vessel enfolding", which ensures the margin for a deep lesion. After anatomical resection, the detection of GGOs is also a challenge without locating, thus our approach is to anatomically dissect and trace the reference vessel, which is selected from the preoperative $3 \mathrm{D}$ reconstruction. The factual foundation of our approach is that the relative position of nodule and adjacent vessels is fixed regardless of whether the lung is in expansion or collapse. In our experience, the pulmonary artery is the best choice of reference vessel, as it has a thicker wall and higher strength than others. The bronchus is rarely selected because it always stays in the center of the conical segment, surrounded by arteries and veins. Selecting a relatively superficial vessel simplifies the tracing procedure. Besides, to ensure that we are tracing the exact reference vessel, repetitious comparisons between the actual anatomical structure and $3 \mathrm{D}$ reconstruction simulation are indispensable. With the popularization of $3 \mathrm{D}$ reconstruction and the extensive and skilled application of precise anatomical segmentectomy, our approach could be widely applied for invisible and impalpable GGO lesions that were not inappropriate for preoperative localization techniques. Our suggestion to resect and detect the invisible and impalpable GGO lesions are as follows, for peripheral GGO, wide wedge resection after preoperative localization is the first choice. While for the lesions that localization techniques were infeasible or inappropriate or/and deep in the parenchyma, precise segmentectomy combining with our localization-independent approach would be the preferred scheme.

To sum up, based on the two cases, we have introduced a new and easily implemented approach to detect invisible and impalpable GGOs in the in vitro surgical specimen with no need for locating, namely dissection and tracing a reference vessel. Our approach, that of combining precise anatomical segmentectomy under the guidance of $3 \mathrm{D}$, is a feasible and alternative scheme, and occasional remedy, for GGOs resection.

\section{Acknowledgments}

Funding: This work was supported by the Key Research and Development Program of Hunan Province, China (2018SK21215).

\section{Footnote}

Reporting Checklist: The authors have completed the CARE reporting checklist. Available at https://dx.doi. org/10.21037/atm-21-4966

Conflicts of Interest: All authors have completed the ICMJE uniform disclosure form (available at https://dx.doi. org/10.21037/atm-21-4966). The authors have no conflicts of interest to declare.

Ethical Statement: The authors are accountable for all aspects of the work in ensuring that questions related to the accuracy or integrity of any part of the work are appropriately investigated and resolved. All procedures performed in this study involving human participants were in accordance with the Declaration of Helsinki (as revised in 2013). The study was approved by Medical Ethics Committee of the Second Xiangya Hospital of Central South University (No. 2019022). Written informed consent was obtained from the patient for publication of this case report and accompanying images. A copy of the written consent is available for review by the editorial office of this journal.

Open Access Statement: This is an Open Access article distributed in accordance with the Creative Commons Attribution-NonCommercial-NoDerivs 4.0 International License (CC BY-NC-ND 4.0), which permits the noncommercial replication and distribution of the article with the strict proviso that no changes or edits are made and the original work is properly cited (including links to both the formal publication through the relevant DOI and the license). See: https://creativecommons.org/ licenses/by-nc-nd/4.0/.

\section{References}

1. Zhang Y, Fu F, Chen H. Management of ground-glass opacities in the lung cancer spectrum. Ann Thorac Surg 2020;110:1796-804.

2. Ye T, Deng L, Xiang J, et al. Predictors of pathologic tumor invasion and prognosis for ground glass opacity featured lung adenocarcinoma. Ann Thorac Surg 2018;106:1682-90. 
3. Zhang Y, Jheon S, Li H, et al. Results of low-dose computed tomography as a regular health examination among Chinese hospital employees. J Thorac Cardiovasc Surg 2020;160:824-31.e4.

4. Chen S, Zhou J, Zhang J, et al. Video-assisted thoracoscopic solitary pulmonary nodule resection after CT-guided hookwire localization: 43 cases report and literature review. Surg Endosc 2011;25:1723-9.

5. Finley RJ, Mayo JR, Grant K, et al. Preoperative computed tomography-guided microcoil localization of small peripheral pulmonary nodules: a prospective randomized controlled trial. J Thorac Cardiovasc Surg 2015;149:26-31.

6. Chen JR, Tseng YH, Lin MW, et al. Safety and efficacy of computed tomography-guided dye localization using patent blue $\mathrm{V}$ for single lung nodule for video-assisted thoracoscopic surgery: a retrospective study. Ann Transl Med 2019;7:28.

7. Zhang B, Peng M, Yu F, et al. A novel technique for preoperative localization of pulmonary nodules using a mixture of tissue adhesive and iohexol under computed tomography guidance: a 140 patient single-center study. Thorac Cancer 2021;12:854-63.

8. Mayo JR, Clifton JC, Powell TI, et al. Lung nodules: CTguided placement of microcoils to direct video-assisted thoracoscopic surgical resection. Radiology 2009;250:576-85.

9. Qiu B, Ji Y, He H, et al. Three-dimensional reconstruction/personalized three-dimensional printed model for thoracoscopic anatomical partial-lobectomy in stage I lung cancer: a retrospective study. Transl Lung Cancer Res 2020;9:1235-46.

10. Yang Q, Xie B, Hu M, et al. Thoracoscopic anatomic pulmonary segmentectomy: a 3-dimensional guided imaging system for lung operations. Interact Cardiovasc Thorac Surg 2016;23:183-9.

11. Chen $\mathrm{L}, \mathrm{Wu} \mathrm{W}$. The main technical points of thoracoscopic anatomical lung segment resection. Zhongguo Fei Ai Za Zhi 2016;19:377-81.

12. Yan TD, Cao C, D'Amico TA, et al. Video-assisted thoracoscopic surgery lobectomy at 20 years: a consensus statement. Eur J Cardiothorac Surg 2014;45:633-9.

13. Hansen HJ, Petersen RH. A video-atlas of video-assisted thoracoscopic lobectomy using a standardized three-port anterior approach. Ann Cardiothorac Surg 2012;1:104.

14. Morgant MC, Pagès PB, Orsini B, et al. Time trends in surgery for lung cancer in France from 2005 to 2012: a nationwide study. Eur Respir J 2015;46:1131-9.

15. Yan TD, Black D, Bannon PG, et al. Systematic review and meta-analysis of randomized and nonrandomized trials on safety and efficacy of video-assisted thoracic surgery lobectomy for early-stage non-small-cell lung cancer. J Clin Oncol 2009;27:2553-62.

16. Falcoz PE, Puyraveau M, Thomas PA, et al. Video-assisted thoracoscopic surgery versus open lobectomy for primary non-small-cell lung cancer: a propensity-matched analysis of outcome from the European Society of Thoracic Surgeon database. Eur J Cardiothorac Surg 2016;49:602-9.

17. Scott AE, Vasilescu DM, Seal KA, et al. Three dimensional imaging of paraffin embedded human lung tissue samples by micro-computed tomography. PLoS One 2015;10:e0126230.

18. Wang Z, Pang L, Tang J, et al. Video-assisted thoracoscopic surgery versus muscle-sparing thoracotomy for non-small cell lung cancer: a systematic review and meta-analysis. BMC Surg 2019;19:144.

19. Yang Y, Mei J, Lin F, et al. Comparison of the shortand long-term outcomes of video-assisted thoracoscopic surgery versus open thoracotomy bronchial sleeve lobectomy for central lung cancer: a retrospective propensity score matched cohort study. Ann Surg Oncol 2020;27:4384-93.

20. Bendixen M, Jørgensen OD, Kronborg C, et al. Postoperative pain and quality of life after lobectomy via video-assisted thoracoscopic surgery or anterolateral thoracotomy for early stage lung cancer: a randomised controlled trial. Lancet Oncol 2016;17:836-44.

21. Aberle DR, Abtin F, Brown K. Computed tomography screening for lung cancer: has it finally arrived? Implications of the national lung screening trial. J Clin Oncol 2013;31:1002-8.

22. Dendo S, Kanazawa S, Ando A, et al. Preoperative localization of small pulmonary lesions with a short hook wire and suture system: experience with 168 procedures. Radiology 2002;225:511-8.

23. Imperatori A, Fontana F, Dominioni L, et al. Videoassisted thoracoscopic resection of lung nodules localized with a hydrogel plug. Interact Cardiovasc Thorac Surg 2019;29:137-43.

24. Shennib H, Bret P. Intraoperative transthoracic ultrasonographic localization of occult lung lesions. Ann Thorac Surg 1993;5 5:767-9.

25. Mack MJ, Gordon MJ, Postma TW, et al. Percutaneous localization of pulmonary nodules for thoracoscopic lung resection. Ann Thorac Surg 1992;53:1123-4.

26. Mullan BF, Stanford W, Barnhart W, et al. Lung nodules: improved wire for CT-guided localization. Radiology 1999;211:561-5. 
27. Park CH, Han K, Hur J, et al. Comparative effectiveness and safety of preoperative lung localization for pulmonary nodules: a systematic review and meta-analysis. Chest 2017;151:316-28.

28. Ichinose J, Kohno T, Fujimori S, et al. Efficacy and complications of computed tomography-guided hook wire localization. Ann Thorac Surg 2013;96:1203-8.

29. Ikeda K, Nomori H, Mori T, et al. Impalpable pulmonary nodules with ground-glass opacity: Success for making pathologic sections with preoperative marking by lipiodol. Chest 2007;131:502-6.

30. Sortini D, Feo CV, Carcoforo P, et al. Thoracoscopic localization techniques for patients with solitary pulmonary nodule and history of malignancy. Ann Thorac Surg 2005;79:258-62; discussion 262.

31. Santambrogio R, Montorsi M, Bianchi P, et al. Intraoperative ultrasound during thoracoscopic procedures for solitary pulmonary nodules. Ann Thorac Surg 1999;68:218-22.

32. Awais O, Reidy MR, Mehta K, et al. Electromagnetic navigation bronchoscopy-guided dye marking for thoracoscopic resection of pulmonary nodules. Ann Thorac Surg 2016;102:223-9.

33. Song JW, Park IK, Bae SY, et al. Electromagnetic navigation bronchoscopy-guided dye marking for localization of pulmonary nodules. Ann Thorac Surg 2021. [Epub ahead of print]. doi: 10.1016/j.athoracsur.2021.05.004.

34. Zhang L, Li M, Li Z, et al. Three-dimensional printing of navigational template in localization of pulmonary nodule: a pilot study. J Thorac Cardiovasc Surg 2017;154:21132119.e7.

35. Chen Z, Han X, Fan G, et al. Preliminary application of three-dimensional printing technique in preoperative localization of meningioma in primary hospitals. J Craniofac Surg 2021;32:1796-9.

36. Koike T, Kitahara A, Sato S, et al. Lobectomy versus segmentectomy in radiologically pure solid smallsized non-small cell lung cancer. Ann Thorac Surg 2016;101:1354-60.

37. Eguchi T, Takasuna K, Kitazawa A, et al. Threedimensional imaging navigation during a lung segmentectomy using an iPad. Eur J Cardiothorac Surg 2012;41:893-7.

38. Wu WB, Xu XF, Wen W, et al. Three-dimensional computed tomography bronchography and angiography in the preoperative evaluation of thoracoscopic segmentectomy and subsegmentectomy. J Thorac Dis 2016;8:S710-5.
39. Kato H, Oizumi H, Suzuki J, et al. Thoracoscopic anatomical lung segmentectomy using $3 \mathrm{D}$ computed tomography simulation without tumour markings for nonpalpable and non-visualized small lung nodules. Interact Cardiovasc Thorac Surg 2017;25:434-41.

40. Xue L, Fan H, Shi W, et al. Preoperative 3-dimensional computed tomography lung simulation before video-assisted thoracoscopic anatomic segmentectomy for ground glass opacity in lung. J Thorac Dis 2018;10:6598-605.

41. Chan EG, Landreneau JR, Schuchert MJ, et al. Preoperative (3-dimensional) computed tomography lung reconstruction before anatomic segmentectomy or lobectomy for stage I non-small cell lung cancer. J Thorac Cardiovasc Surg 2015;150:523-8.

42. El-Sherif A, Fernando HC, Santos R, et al. Margin and local recurrence after sublobar resection of non-small cell lung cancer. Ann Surg Oncol 2007;14:2400-5.

43. Sawabata N, Ohta M, Matsumura A, et al. Optimal distance of malignant negative margin in excision of nonsmall cell lung cancer: a multicenter prospective study. Ann Thorac Surg 2004;77:415-20.

44. Koike T, Koike T, Yoshiya K, et al. Risk factor analysis of locoregional recurrence after sublobar resection in patients with clinical stage IA non-small cell lung cancer. J Thorac Cardiovasc Surg 2013;146:372-8.

45. Wu X, Li T, Zhang C, et al. Comparison of perioperative outcomes between precise and routine segmentectomy for patients with early-stage lung cancer presenting as groundglass opacities: a propensity score-matched study. Front Oncol 2021;11:661821.

46. Ji Y, Zhang T, Yang L, et al. The effectiveness of threedimensional reconstruction in the localization of multiple nodules in lung specimens: a prospective cohort study. Transl Lung Cancer Res 2021;10:1474-83.

47. Li M, Shen G, Gao F, et al. CT-guided fine-needle localization of ground-glass nodules in re-aerated lung specimens: localization of solitary small nodules or multiple nodules within the same lobe. Diagn Interv Radiol 2015;21:391-6.

(English Language Editor: J. Jones)

Cite this article as: Qing B, Xia Z, Wang W, Gu L, Chen H, Yuan Y. A localization-independent approach for invisible and impalpable ground-glass opacity nodules detection in an in vitro lung specimen: two case reports. Ann Transl Med 2021;9(20):1602. doi: 10.21037/atm-21-4966 